\title{
Restauración ecológica de disturbios antrópicos presentes en la zona alto andina
}

\author{
Ecological restoration of anthropic disturbances in the high Andean zone
}

\section{Pedro Pablo Bacca A. ${ }^{1}$; Diana Lucía Burbano M. ${ }^{2}$}

\footnotetext{
${ }^{1}$ Docente, I.AF. M.Sc. Ingeniería Ambiental. Universidad de Nariño. Pasto, Colombia, pedroingeagro@gmail.com.

${ }^{2}$ Bióloga, Biofuturo, Pasto, Colombia, dbiologia@gmail.com.
}

Citar: Bacca, P. \& Burbano, D. (2018). Restauración ecológica de disturbios antrópicos presentes en la zona alto andina. Revista de Ciencias Agrícolas. 35(2): 36-50. doi:http://dx.doi.org/10.22267/rcia.183502.90

Recibido: mayo $262016 . \quad$ Aceptado: febrero 23 de 2018.

\begin{abstract}
RESUMEN
La restauración ecológica es reconocida globalmente como una actividad importante en los esfuerzos de conservación, revertimiento de la degradación ambiental y moderación del cambio climático. A pesar de ser una disciplina joven, la restauración ecológica, ha avanzado rápidamente en sus fundamentos científicos y sus métodos teórico-prácticos. El objetivo de este trabajo fue evaluar la metodología de restauración ecológica con el fin de identificar las especies nativas más importantes para este proceso en Bosque Alto Andino. Para ello, inicialmente se evaluaron las diferentes zonas de disturbios teniendo en cuenta las metodologías, se establecieron las especies potenciales de restauración ecológica. El estudio se realizó en tres tipos de uso de suelo como son bosque, uso agrícola y uso pecuario por medio de las variables altura, cobertura y número de ramas de las especies de plantas. Para cada uno de los usos de suelo se realizó el Analisis de Componentes Principales, del cual se obtuvo como resultado una correlación entre la altura y el uso de suelo bosque y entre cobertura y número de ramas para el uso pecuario; sin embargo, el uso agrícola no presentó relación entre las variables lo que lleva a concluir que este tipo de suelo ha sido sometido a otros tipos de disturbios en épocas pasadas. Se concluyó que Oreopanax floribundum es una alternativa para iniciar procesos de restauración para estos tipos de disturbio.
\end{abstract}

Palabras clave: conservación, disturbio, biodiversidad, flora nativa, uso de suelo. 


\begin{abstract}
Restoration ecology is globally well-known as an important activity in efforts to preserve and revert the Environmental degradation as well as to moderate the climate change. Despite being a young discipline, the Restoration ecology has rapidly advanced on its Scientific Foundations and theoreticalpractical methods. The aim of this paperwork was to evaluate the methodology of the restoration ecology in order to identify the most important native species in the upper Andean forest. Initially it was carried out an evaluation of the different areas of disturbances according to the methodologies, also it was stablished the potential species to be ecologically preserved. This study was achieved in three different type of grounds and variables such as height, coverage and number of the branches of the plant species were studied. For the ground study was fulfilled an analysis of the main components which showed a correlation among the height, the coverage and number of the branches of the plants for agricultural usage; however, this agricultural usage of the plants didn't show a relationship among the variables, thus we concluded that the ground was under other type of disturbances in the past. Finally we noticed that Oreopanax floribundum is an alternative to initiate restoring processes for these types of disturbance.
\end{abstract}

Key words: Preservation, disturbance, biodiversity, native flora, usage of the ground

\section{INTRODUCCIÓN}

La degradación de los ecosistemas en el mundo ha acelerado la crisis ambiental debido a la reducción de los servicios ambientales ofrecidos por los ecosistemas, talescomo producción deagua,fijación de $\mathrm{CO}_{2}$, productividad del suelo, biodiversidad, coberturas que previenen erosión, entre otros. Las tasas de destrucción, aumentan debido a prácticas agrícolas, ganaderas, industriales y de explotación no sostenibles (Vargas, 2011).

Por lo tanto, cuando los ecosistemas están muy degradados o destruidos, han perdido sus mecanismos de regeneración y en consecuencia, es necesario adelantar estrategías de restauración ecológica. Ésta implica, que con la intervención humana, se asista al ecosistema para garantizar el desarrollo de los procesos de recuperación y superar los tensionantes que impiden la regeneración (Vargas et al., 2010). En algunos casos, los daños ocasionados al ecosistema son severos debido a que las perturbaciones son intensas o se prolongan por demasiado tiempo, de manera que afectan seriamente los procesos sucesionales, que disminuyen la habilidad del ecosistema para recuperarse en forma natural (Bradshaw, 1987). En estos casos, cuando la perturbación es intensa y frecuentemente la re- moción de la causa perturbadora puede no bastar para lograr su recuperación y las propiedades del ecosistema pueden verse seriamente modificadas o afectadas, incluso pueden causar deficiencias en la disponibilidad de agua, pérdida de la capa de suelo con el subsecuente detrimento en nutrientes y materia orgánica (Whisenant et al., 1995) lo que provoca la degradación del ecosistema.

En los disturbios extremos la explotación ha sido persistente durante mucho tiempo ocasionando en el suelo una intensiva extracción de nutrientes, erosión y degradación (Rehm, 2009). Como solución a este problema se plantea el manejo del ecosistema a través de la restauración ecológica, la cual implica la interacción de las plantas con su entorno cuyo fin es mejorar la calidad integral de los ecosistemas y su expresión como paisaje, que constituye una herramienta primordial dentro de los planes de conservación y gestión de especies y espacios. El término restauración integran dos objetivos que persiguen las actuaciones, las cuales son: a) orientar el dinamismo espontáneo del sistema hacia un determinado mosaico de comunidades de organismos y b) acelerar el proceso que espontáneamente habría tardado en suceder un tiempo más dilatado o no habría ocurrido por sí mismo. 
En la zona de Bosque Alto Andino se evidencian estas perturbaciones al ecosistema, que deterioran los servicios ambientales que éste ofrece a las comunidades locales, sin embargo, éstas perturbaciones tienen un origen antrópico debido a la explotación de los recursos naturales, por esta razón, los tipos de disturbios frecuentemente encontrados en estos lugares son la extracción de especies forestales, quemas, plantaciones de especies exóticas, zonas con pastoreo y agricultura intensiva; estas dos últimas, se tratarán en esta investigación debido a que influyen principalmente en la disminución de los límites del Bosque Alto Andino debido a que generan ingresos a la comunidad local y son aplicados sin ningún tipo de manejo (Etter, 1998; Kattan, 2002; Rodríguez y Van Hoof, 2004).

Esta investigación se orientó hacia la evaluación de una metodología de restauración ecológica adaptada a la zona de Bosque Alto Andino con disturbios antrópicos de tipo agrícola o pecuario. Con el fin de iniciar la restauración con la recuperación del suelo se adicionó fertilizante orgánico, por medio de la implementación de parcelas demostrativas de restauración ecológica con especies nativas de bosque Alto Andino en la Granja Experimental Botana de la Universidad de Nariño.

\section{MATERIALES Y MÉTODOS}

Localización. La investigación se llevó a cabo en la Granja Experimental Botana de la Universidad de Nariño, ubicada en la vereda Botana, corregimiento de Catambuco, municipio de Pasto, Nariño, con coordenadas $01^{\circ} 09^{\prime} 12^{\prime \prime}$ LN y $77^{\circ} 18^{\prime} 31^{\prime \prime}$ LO, a una altura de $2.820 \mathrm{msnm}$, con temperatura promedio de $13^{\circ} \mathrm{C}$, precipitación media anual de $967 \mathrm{~mm}$, humedad relativa del 75\% (Lagos et al., 2001). Según la clasificación de zonas de vida de Holdridge (1990) pertenece a un bosque húmedo montano bajo (bh-MB).

Trabajo de campo. Para esta investigación se modificó la metodología de Vargas et al. (2010) por medio de la aplicación de un tratamiento para el suelo, como es la adición de fertilizante orgánico en las zonas con disturbio agrícola o pecuario en bosque Alto Andino esto con el fin de implementar parcelas demostrativas con especies nativas.

\section{Fase diagnóstica del ecosistema}

Ecosistema de referencia. Este tipo de ecosistema hace referencia a la zona de bosque que ha sido conservada la cual permitió realizar una reconstrucción histórica del ecosistema a restaurar; extensión, especies importantes, época en la que aparecieron los diferentes usos de suelo.

Estado actual del ecosistema. El diagnostico se realizó por medio de visitas a conocedores de la zona a restaurar, los cuales por medio del dialogo aportaron en la identificación de los tipos de disturbios presentes en el Bosque Alto Andino. Disturbios referentes a intervención atropica a causa de uso agrícola y pecuario que visualmente se pudo corroborar en campo.

\section{Fase potencial de regeneración}

Esta fase tuvo en cuenta el potencial de regeneración. Se cuantificó la disponibilidad de especies en la región con base en información secundaría, así mismo, se estableció su ubicación, su etapa sucesional y abundancia, con el fin de obtener una línea base para iniciar el trabajo en campo. Seguidamente, se aplicó la metodología de transectos de Gentry (1991), donde se realizaron 10 transectos de 50 x $2 \mathrm{~m}$ para árboles y arbustos y de 1 x $1 \mathrm{~m}$ para herbáceas, de los cuales se registraron datos ecológicos, descripción del hábitat y características ambientales de la zona.

Se evaluaron variables ecológicas como: densidad (conteo del número de individuos en cada una de las unidades muestréales), frecuencia (cálculo del número de parcelas en donde se encontraron la especie y número total de unidades muestréales), altura, diámetro a la altura del pecho y cobertura (estas dos últimas variables para plantas con una altura mayor a dos metros). 
Trabajo de herbario. Las especies colectadas y previamente prensadas se sometieron al proceso de secado e identificación en el herbario PSO de la Universidad de Nariño.

En esta fase se tuvo una aproximación a las especies que potencialmente pueden ser utilizadas en experimentos de restauración.

\section{Fase experimental}

Para la fase experimental se tuvo en cuenta los siguientes pasos:

Selección de especies. Del listado de especies y sus trayectorias sucesionales registrado en el potencial de regeneración, se seleccionaron las especies ecológicamente más importantes teniendo en cuenta, además, una escala de atributos o rasgos de historia de vida que pueden ser útiles en los sitios que se van a restaurar.
Propagación y manejo de especies. Con la ayuda de personal previamente capacitado en la recolección de especies nativas se obtuvo el material vegetal necesario para propagarlo en el vivero de la Granja Experimental Botana. Los requerimientos para la recolección del material vegetal fueron: plantas vigorosas con un tamaño entre 20 y $50 \mathrm{~cm}$ y totalmente enraizadas, provenientes de bordes de caminos y potreros, para ser sembradas en bolsas de polietileno de media libra.

Selección de los sitios para restaurar. En este ensayo se tuvieron en cuenta tres tipos de disturbio (Figura 1) a los cuales se les añadió biofertilizante-abono Supermagro: Bosque de referencia, disturbio Agrícola, disturbio pecuario donde se medió variables como: altura y cobertura número de ramas.

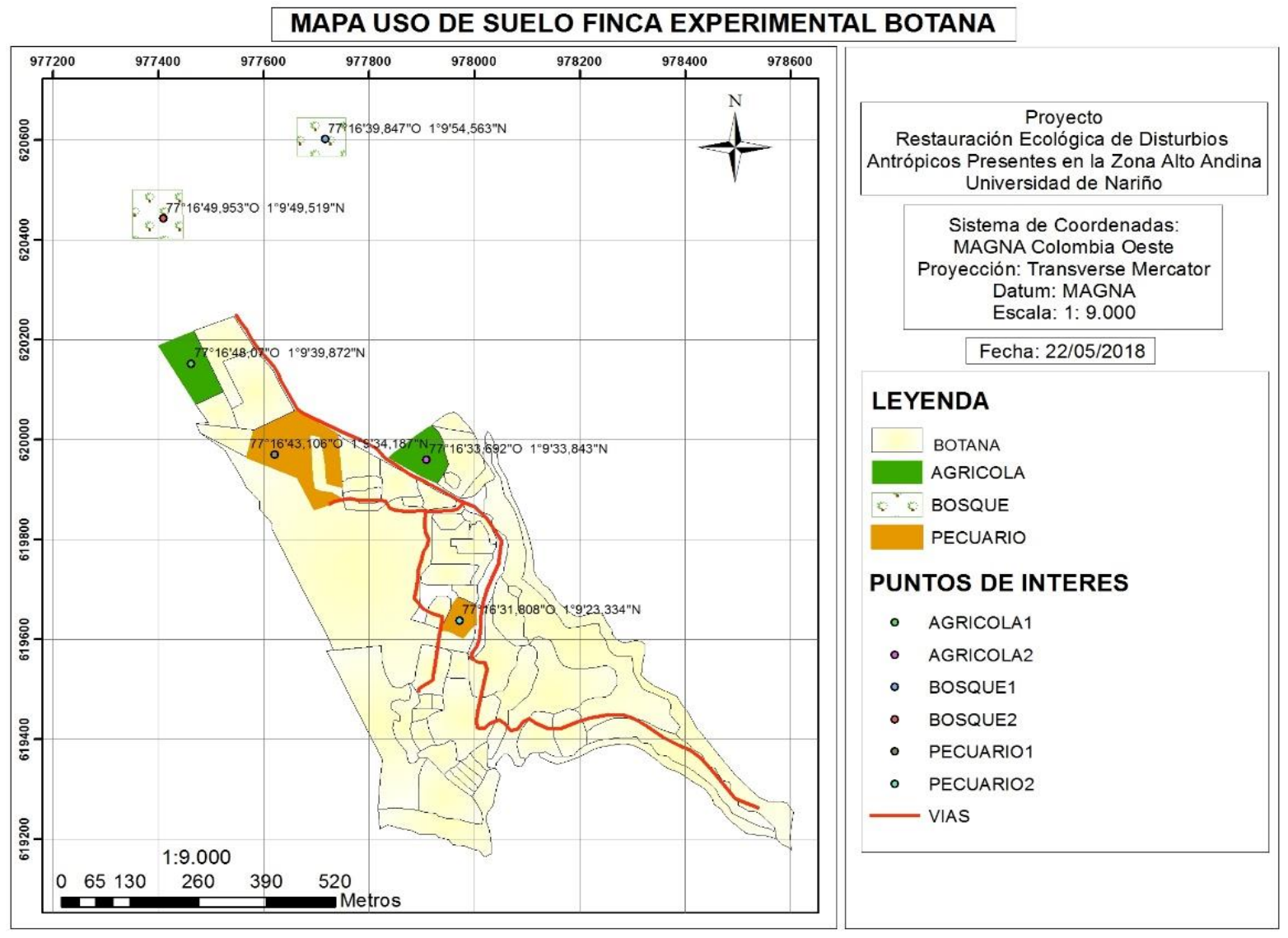

Figura 1. Mapa de uso de suelo de la Granja Experimental Botana. 
En tres parcelas demostrativas se implementaron cuatro núcleos de restauración. Los sitios a restaurar fueron delimitados con polisombra con el propósito de brindar condiciones micro climáticas óptimas para el desarrollo de las plántulas ecológicamente importantes, tomadas de la selección de especies, evitando su desecación por efecto del viento. Estas especies con habito de crecimiento tipo herbáceo y arbustivos se muestran en la Tabla 1 y Tabla 2, esta información fue una parte del inventario florístico que se realizó en la zona de estudio.

Tabla 1. Inventario florístico del Bosque Alto Andino perteneciente a la Granja Experimental Botana de la Universidad de Nariño, corregimiento de Catambuco, Municipio de Pasto.

\begin{tabular}{ll}
\hline \multicolumn{1}{c}{ Especie } & Hábito de crecimiento \\
\hline Cortaderia selloana (Schult. \& Schult. f.) & Herbácea \\
Cyathea caracasana (Klotzsch) Domin & Herbácea \\
Gynerium sagittatum (Aubl.) P. Beauv. & Herbácea \\
Oreopanax floribundum (Kunth) & Herbácea \\
Papaver somniferum L. & Herbácea \\
Phragmites australis (Cav.). Trin. ex Steudel & Herbácea \\
Rubus ulmifolius Schott & Herbácea \\
Sanseviera trifasciata Prain & Herbácea \\
Saurauia pruinosa R.E. Schult. & Herbácea \\
Tanacetum balsamita L. & Herbácea \\
Viburnum triphyllum Benth. & Herbácea \\
Luma apiculata (DC.) Burret & Arbórea y arbustiva \\
Miconia sp. & Arbórea y arbustiva \\
Morella pubescens & Arbórea y arbustiva \\
Prunus serótina Ehrh. & Arbórea y arbustiva \\
Teracera volúbilis L. & Arbórea y arbustiva \\
Tipuana tipu (Benth.) Kuntze & Arbórea y arbustiva \\
Viburnum triphyllum Benth & Arbórea y arbustiva \\
\hline
\end{tabular}

Tabla 2. Especies potenciales para la restauración.

\begin{tabular}{lc}
\hline \multicolumn{1}{c}{ Especie } & Hábito de crecimiento \\
\hline Anthurium andreanum & Herbácea \\
Cythea caracasana & Arbóreo \\
Miconia sp. & Arbóreo \\
Myrica pubescens & Arbustivo \\
Oreopanax floribundus & Arbóreo \\
Sanseviera trifasciata & Herbácea \\
Tibouchina lepidota & Arbustivo \\
\hline
\end{tabular}


El tamaño de los nucleos de restauración y la distancia entre ellos varió de acuerdo con las dimensiones del disturbio de establecimiento, pero en general, cada núcleo fue delimitado dentro de un diámetro de cinco metros lineales y se ubicaron a una distancia de dos metros unos de otros.

Forma de siembra. Se sembraron 100 individuos de especies nativas en cada núcleo, en forma circular formando a su vez tres círculos.

Fase de monitoreo. Se llevó un registro mensual de altura en $\mathrm{cm}$, cobertura en porcentaje y número de ramas el número de especies sembradas fue del 70\% para herbáceas y 30\% para árboles.

\section{Análisis Estadístico}

Se utilizó el Análisis de Componentes Principales (ACP) (Cantillo et al., 2004), con el fin de observar que variables influyen en la restauración ecológica según el tipo de disturbio.

Esta técnica multivariada permite el análisis de las unidades experimentales tales como las especies, las parcelas de experimentación y las zonas de disturbio, en forma simultánea, a partir de la medición de una serie de características entre las que se cuentan sus tratamientos o variables (Cantillo et al., 2004).

\section{RESULTADOS Y DISCUSIÓN}

Fase de diagnostico del ecosistema. Los datos obtenidos en campo permitieron reconocer que el ecosistema de referencia tiene un área de 87ha de bosque, los cuales encuentran en estado de conservación desde hace 50 años (Tabla 3). De acuerdo con Walker y Del Moral (2003), la conservación de ecosistemas permite la presencia de arbustales que introducen modificaciones micro-ambientales favorables para los procesos edáficos, el desarrollo de una entomofauna edáfica más variada, mejores condiciones para percheo y nidación de aves y como refugio de anfibios y otros elementos de la fauna.

En esta zona de bosque se encuentran coberturas antropizadas, las cuales se refieren a la sumatoria de las áreas transformadas por actividades en este caso productivas (áreas agrícolas heterogéneas con cultivos de pasto y papa principalmente, cultivos transitorios, pastosy zonas de actividad minera) (IAvH, 2012). Por lo tanto, se identificó la permanencia del uso pecuario y agrícola (Figura $1)$, los cuales, han estado en constante actividad hace varios años, responsables de los disturbios en la zona de estudio.

Tabla 3. Usos de suelo en la Granja Experimental Botana de la Universidad de Nariño, Corregimiento de Catambuco, Municipio de Pasto, Nariño.

\begin{tabular}{|c|c|c|c|c|}
\hline \multirow{2}{*}{$\begin{array}{l}\text { Tipo de uso de } \\
\text { suelo }\end{array}$} & \multicolumn{2}{|c|}{ Coordenadas } & \multirow[b]{2}{*}{ Altitud } & \multirow[b]{2}{*}{ Observaciones } \\
\hline & $\mathbf{N}$ & $\mathbf{W}$ & & \\
\hline Bosque & $01^{\circ 9} 54,563^{\prime \prime} \mathrm{N}$ & $077^{\prime} 16^{\prime} 39,847^{\prime \prime} 0$ & $2810 \mathrm{msnm}$ & $\begin{array}{l}\text { Se encuentra en protección } \\
\text { aproximadamente hace } 50 \\
\text { años. }\end{array}$ \\
\hline Pecuario & $01^{\circ} 09^{\prime} 23,334^{\prime \prime} \mathrm{N}$ & 077ํ16’31,808”0 & $2790 \mathrm{msnm}$ & $\begin{array}{l}\text { Constantemente está en } \\
\text { pastoreo de ganado. }\end{array}$ \\
\hline Agrícola & $01^{\circ} 9^{\prime} 39,872^{\prime \prime} \mathrm{N}$ & $077^{\circ} 16^{\prime} 48,07^{\prime \prime} 0$ & $2790 \mathrm{msnm}$ & $\begin{array}{l}\text { El cultivo de papa se presenta } \\
\text { como cultivo permanente }\end{array}$ \\
\hline
\end{tabular}


Al respecto, Barrera et al. (2004), Barrera y Valdés (2007), afirman que debido al uso agropecuario intenso y continuo en las microcuencas hay una transformación y degradación tan fuerte, que hoy es posible observar áreas desprovistas de cobertura vegetal y de suelo con estados muy avanzados de degradación que hacen muy difícil pensar en el restablecimiento de la estructura y función de sus ecosistemas situación similar que se presenta en la zona de Bosque Alto Andino de la granja.

Como se puede observar, es evidente que para la gran mayoría de las zonas Andinas que tienen algún nivel de intervención, las áreas categorizadas con actividad agrícola heterogénea son las que mayor impacto tienen en la transformación de coberturas vegetales, en donde las coberturas categorizadas como pastos, representan el principal motor de transformación en dichos ecosistemas de alta montaña seguido por la actividad minera (FierroMorales, 2012).

Es fundamental reconocer que la pérdida de funciones ecológicas y de servicios ecosistémicos disminuyen el rendimiento de los sistemas de producción por la pérdida de fertilidad en los suelos (Jaimes y Sarmiento, 2002). En la granja de Botana, el uso de suelo con mayor extensión, corresponde a bosque, seguida de uso pecuario y agrícola (Figura 2), lo cual a su vez, brinda una reseña de los tipos de disturbio que se presentan en el lugar y que merecen un manejo. Estos disturbios, afectan de forma drástica todos los componentes y las condiciones micro, meso y macro climáticas del ecosistema, que afectan la composición, estructura y función de la biota y del suelo, así como la dinámica hídrica, los flujos de nutrientes y la capacidad regenerativa natural de los ecosistemas, compactación del suelo, lixiviación de nutrientes y pérdida de materia orgánica, factores que pueden generar comunidades propias de sucesiones secundarias o desviadas (ecosistemas degradados), por lo tanto, se consideran los disturbios antrópicos ocasionados por los sistemas productivos agrícolas y pecuarios como los causantes de los daños en el suelo (Plan Nacional de Restauración, 2015).

Adicionalmente, a la presencia de sistemas productivos como se ha mencionado en estudios anteriores para zonas de páramos que consecuentemente tiene las mismas historias de disturbios, es posible evaluar el estado de conservación de éstos y sus prioridades de restauración a través del tamaño y la forma de las islas (parches de vegetación). En términos generales, con base en los tamaños se evalúa la extensión de cada cobertura, si están conservados su extensión será mayor, pero si están intervenidos o fuertemente intervenidos, su extensión será menor con mayor cantidad de islas pequeñas separadas entre sí y con coberturas antrópicas (Cabrera y Ramírez, 2014).

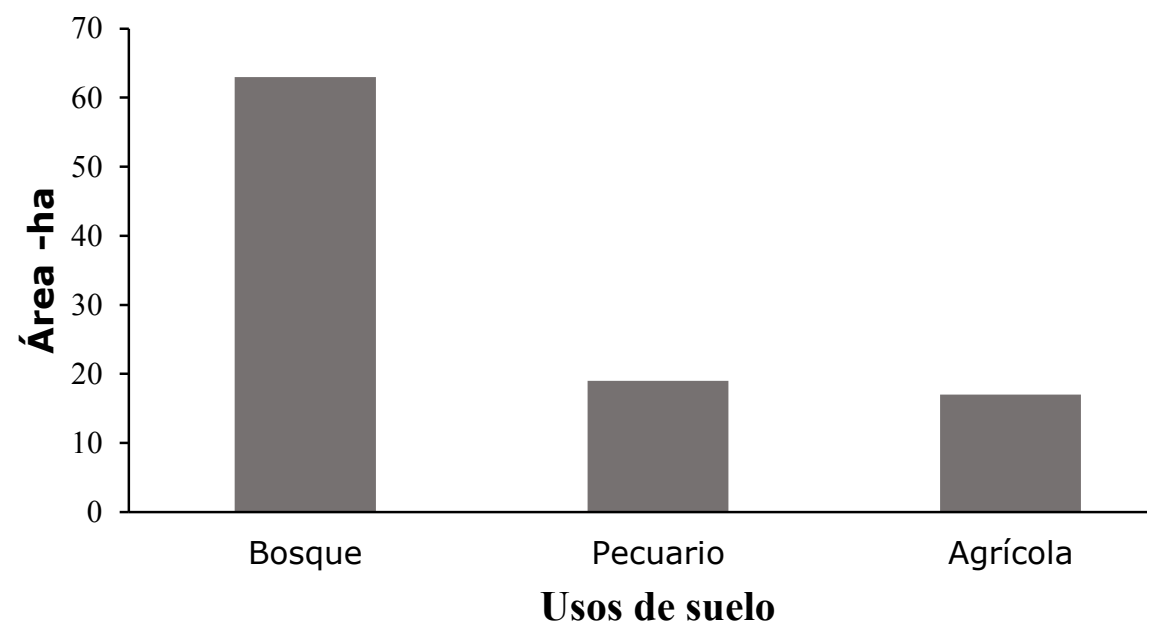

Figura 2. Distribucion y área de usos de suelo en la Granja Exprimental BotanaUniversidad de Nariño. 
Con respecto a las condiciones en las que se hallaban los tres tipos de uso de suelo en la granja, se observó que los suelos con uso agrícola y pecuario presentaron un régimen de implicación alto con relación a los disturbios encontrados, así mismo, presentaban mayor contaminación por el uso desmedido de agroquímicos y erosión, y menor diversidad de especies en comparación con el uso de suelo de bosque lo que las caracteriza como zonas degradadas y conlleva a tenerlas en cuenta para iniciar el proceso de restauración como lo recomienda Vargas et al. (2007). Cabe mencionar que estos tipos de suelo tienden a crecer, pues según Tejedor et al. (2012) en la región norte y sur de los Andes de Colombia, el patrón de uso de la tierra ha creado un mosaico de hábitats transformados para la expansión de las actividades agrícolas, las plantaciones forestales y pecuarias, y por lo tanto, es necesario buscar mecanismos que promuevan la restauración ecológica efectiva. Además, Bradshaw (1987) y la SER (2004), establecen que la restauración de ecosistemas forestales en zonas degradadas aspira a simular e incluso acelerar los procesos que dirigen la sucesión secundaria, con el objetivo de transformar el ecosistema perturbado en un estado semejante a su condición original, con la recuperación de la estructura y funciones perdidas.

En las zonas de alta montaña existe una dinámica cultural dada por la presencia de comunidades indígenas y campesinas que habitan estas zonas desde hace mucho tiempo (Aranguren y Monasterio, 1997). Gran parte de esta dinámica, está relacionada con los sistemas de producción agrícola que allí se han desarrollado, con sistemas tradicionales de autoconsumo, sistemas con descansos largos y sistemas agro-comerciales muy intensivos en tecnología y capital (Abreu et al., 2009). Las distintas dinámicas de transformación y ocupación, no solo han determinado el nivel de degradación, sino también, han ocasionado una gran diversidad de contextos y paisajes culturales de estas zonas alto andinas (Llambi y Cuesta, 2013).
Fase de potencial de regeneración. Existen umbrales entre algunos estados del ecosistema que impiden su retorno a un estado de menor degradación (Hobbs y Harris, 2001; Bestelmeyer et al., 2003), a menos que se realice algún tipo de manejo; es decir, el sistema presenta unos factores dominantes que impiden o limitan el desarrollo de la sucesión natural en áreas alteradas por disturbios naturales y antrópicos (Aidé y Cavellier 1994, Holl et al., 2000).

Los limitantes ecológicos corresponden a la interacción de los factores bióticos y abióticos, como resultado de los procesos de alteración. Los factores limitantes influyen en las fases más importantes de la vida de las plantas: dispersión, establecimiento y persistencia (Vargas et al., 2007). Las limitaciones a éste proceso en sus diferentes etapas, afectan la riqueza de las especies de la comunidad y la estructura espacial de las poblaciones; además, existen limitantes socioeconómicas que corresponden a los obstáculos sociales, institucionales, de gestión y manejo, así como de los recursos económicos que están asociados al uso de un ecosistema en particular, que dificultan e impiden las acciones de restauración.

El inventario florístico que permitió conocer las especies potenciales para realizar procesos de restauración, el cual mostró once especies de herbáceas y siete especies entre árboles y arbustos, con posible potencial para ser utilizados en estos procesos (Tabla 1). Según Ramírez-Marcial (2014), reconocer las especies vegetales pioneras y el papel que cumplen en los procesos de conservación y restauración puede marcar la diferencia entre su éxito o fracaso, aunque encontrarlas sea un poco complicado dado las diferentes perturbaciones presentes en la zona, dentro de las cuales, de acuerdo con Constantino et al. (2006), se reducen las poblaciones de especies a causa de la deforestación.

Los índices de Simpson y Shannon - Wiener y la abundancia de cada una de las especies de herbá- 
ceas, árboles y arbustos (Figuras 3 y 4) (Tabla 4), indican que especies como $V$. triphyllum, C. selloana, C. caracasana, S. trifasciata, T. volubilis, Miconia $s p$, M. pubescens, T. tipu y V. triphyllum son potenciales y pioneras para la restauración debido a sus condiciones morfológicas y contribución ecológica (Ramírez-Marcial, 2014). Según Vargas y Ramírez
(2014), las especies pioneras intermedias constituyen ese grupo de árboles y algunos arbustos de rápido crecimiento que pueden permanecer en los ecosistemas por un tiempo largo y son claves en los procesos de sucesión, la oferta de recursos para la fauna y la generación de oportunidades para otras especies.

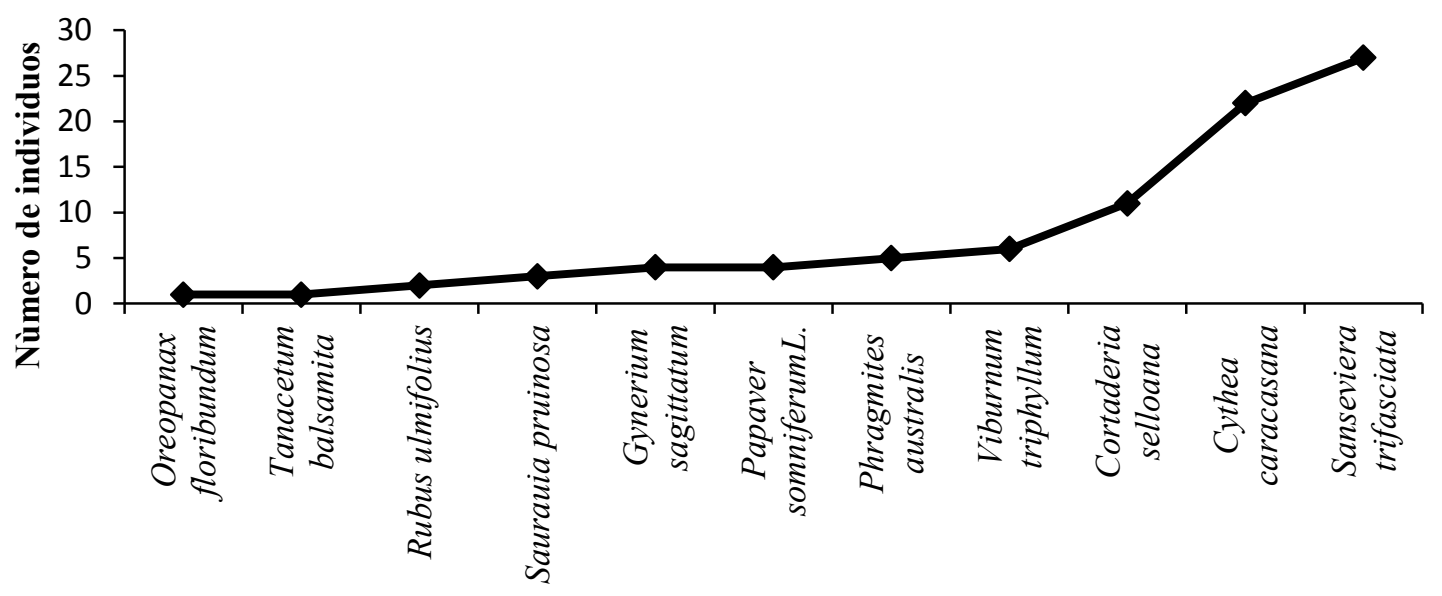

Figura 3. Abundancia estrato herbáceo.

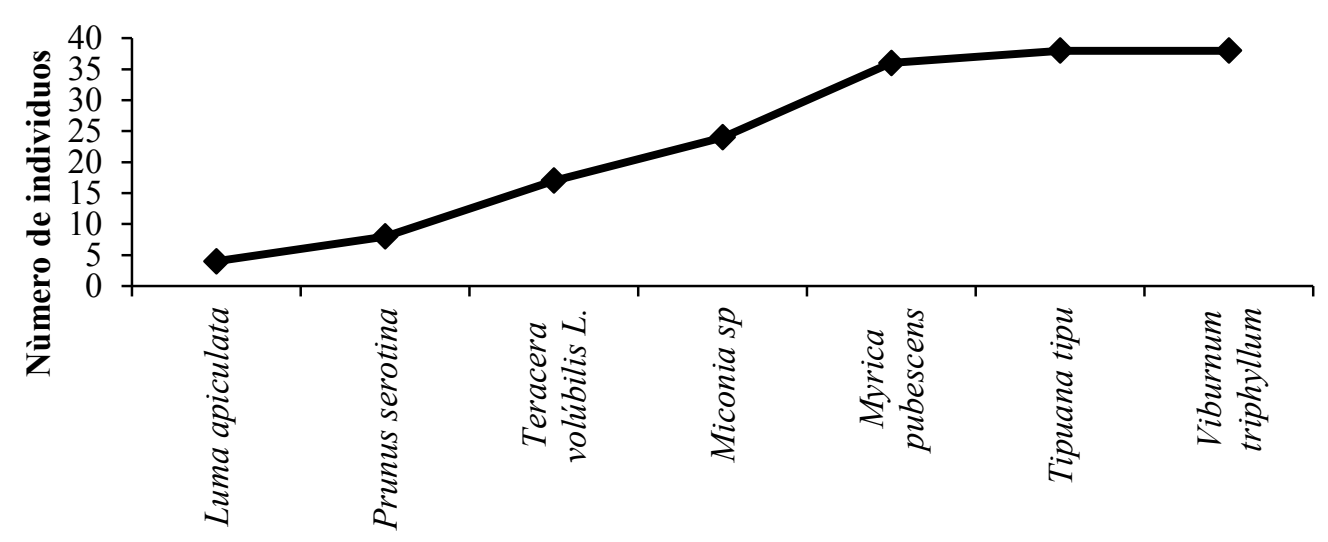

Figura 4. Abundancia estrato arbóreo y arbustivo.

Tabla 4. Índices de diversidad para herbáceas, árboles y arbustos.

\begin{tabular}{lrl}
\hline \multicolumn{1}{c}{ Índices de diversidad } & Simpson & Shannon-Wiener \\
\hline Herbáceas & 0,12323764 & 1,05964496 \\
Árboles y arbustos & 0,17745916 & 0,85643296 \\
\hline
\end{tabular}


La cantidad de individuos que se encontraron en el ecosistema, fue alta (Figura 4), por lo tanto, no han sido afectadas por la extracción para continuar con la siguiente fase de la restauración. De acuerdo con Vargas et al. (2010), el éxito de proyectos de restauración, depende de la selección de especies. Por consiguiente, del listado de especies y sus trayectorias sucesionales registrado en el potencial de regeneración, se deben seleccionar aquellas más importantes bajo una escala de atributos o rasgos de historia de vida que pueden ser útiles en los sitios que se van a restaurar. En esta fase es necesario combinar el conocimiento de la gente, el conocimiento de expertos locales y científicos.

Fase de experimentación. Normalmente, las razones por las cuáles un ecosistema debe ser restaurado son muy diversas y en ocasiones poco comprendidas (Clewell y Aronson, 2006), debido a las fallidas reforestaciones de los páramos con especies exóticas. Por lo tanto, la restauración ecológica en Colombia permite vincular la restauración a un principio de realidad socio-económico donde se garantiza la apropiación local de las actividades de restauración (Blignaut et al., 2013, Aronson et al., 2006).

Para la tercera fase se llegó a un consenso entre los conocedores locales y la información sobre las especies potenciales para la restauración que arrojó el inventario florístico junto con los datos de abundancia e índices calculados ( Tabla 1). Este consenso tuvo lugar debido a la importancia del conocimiento local frente al desarrollo de la restauración, pues las poblaciones humanas, son quienes conocen el ecosistema y quienes están encargadas de cuidar y valorar los servicios que él ofrece. De acuerdo con Farrington y Martin (1988), el saber local es el acervo de conocimientos, creencias y costumbres consistentes entre sí y lógicos para quienes los comparten. A su vez Grenier (1998), menciona que está constituido por saberes y percepciones únicos para una cultura 0 una sociedad dada. Al respecto, Johnson (1992), establece que este conocimiento sobre el medio ambiente es acumulativo y dinámico, basado en la experiencia de generaciones pasadas y adaptado a los nuevos cambios tecnológicos y socioeconómicos del presente.

Debido a las prácticas de agricultura intensiva y la conversión a sistemas de ganadería extensiva, los cuales se convierten en motores de cambio de la zona de alta montaña en un área propensa a sufrir procesos de extinción local, ya que la pérdida de diversidad involucra la desaparición de especies endémicas, así como los cambios de coberturas vegetales y la introducción de especies exóticas en monocultivos, por tal razón, es importante conocer las especies adecuadas para iniciar los procesos de restauración (Cabrera y Ramirez, 2014).

Se identificaron $A$. andreanum y S. trifasciata como especies principales o pioneras para el proceso de restauración, las cuales presentaron un hábito de crecimiento herbáceo. Esto permite establecer un referente sobre las especies a utilizar en futuros ensayos de restauración, cuyos esfuerzos actuales en la zona son escasos. De igual forma, al tener una contextualización de las especies pioneras para la restauración, permitirá dar el primer paso hacia la subsistencia y manejo sostenible de ecosistemas, los que a medida que aumenta el uso de sus recursos, será más frecuente encontrarse con casos críticos que requieran de restauración ecológica (Machlis, 1993). Al respecto, Finegan (1993), indica que los procesos de sucesión secundaria son poco exitosos, debido a que el fenómeno de deterioro antropogénico es relativamente reciente y los mecanismos evolutivos que permitirían la adaptación de ciertas especies a tales condiciones, aún no han operado. Según UICN (1999), el restablecimiento de la producción de los suelos degradados debe ser necesariamente la orientación principal de la labor de restauración en muchos lugares, especialmente en las zonas más pobres y más densamente pobladas.

Fase de Monitoreo. Se evaluó altura, cobertura y número de ramas en cada uno de los usos de suelo. Se aplicó una Prueba de Componentes Principales o PC (Tabla 5, Figura 5) la cual indica una estrecha 
relación entre los usos de suelo agrícola y pecuario si se tiene en cuenta variables como cobertura y número de ramas. Sin embargo, se encontró mayor relación entre dichas variables y el uso pecuario, indicando que, a pesar de los altos daños causados por la ganadería, la restauración de este uso de suelo es más eficaz en la zona de estudio.
Tabla 5. Análisis de Componentes Principales

\begin{tabular}{ccc}
\hline PC & Eigenvalor & \% varianza \\
\hline 1 & 83,93 & 89,43 \\
2 & 9,920 & 10,57 \\
3 & 3,267 & $3,48 \times 10^{-31}$ \\
\hline
\end{tabular}

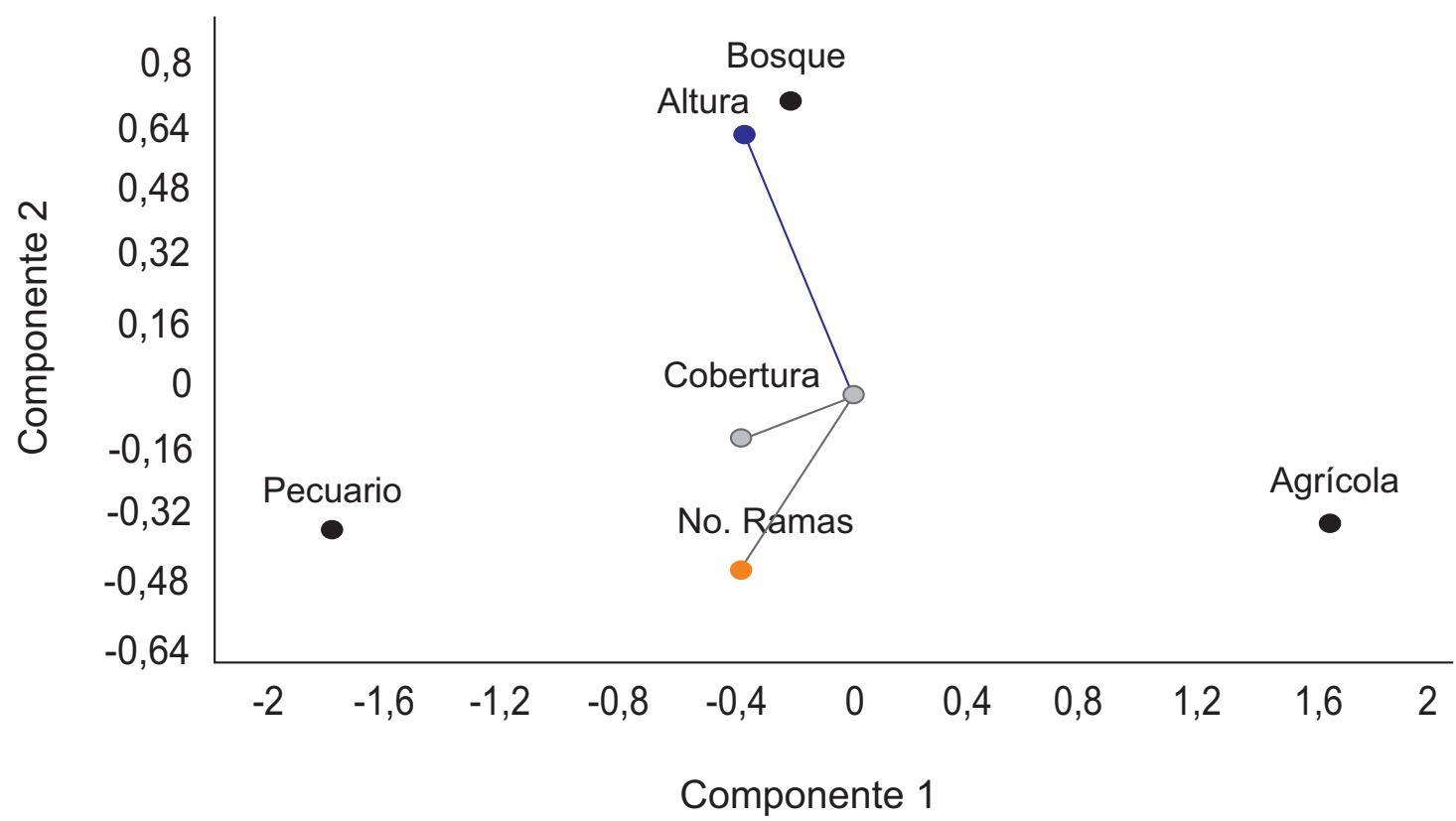

Figura 5. Diagrama Análisis de Componentes Principales

Por otro lado, en el uso de suelo con bosque, se encontró una mayor relación con la variable altura de las plantas, debido a la respuesta de las plantas a la búsqueda de la luz o por la competencia de nutrientes del suelo para aumentar su tamaño y conseguir la supervivencia. Debido a las causas anteriores se producen los diferentes estratos dentro del ecosistema de bosque. Acorde con esto, Terborgh (1992), menciona que la estratificación de los bosques está relacionada con el grado de iluminación del mismo, de tal forma que bajo unas condiciones particulares de luz, se agrupa una determinada cantidad de individuos pertenecientes a especies con similares requerimientos lumínicos. La estratificación se da gracias al proceso estructural del bosque donde la poca o nula incidencia de luz incita a respuesta de la panta para asegurar su supervivencia (Otavo, 1994).

Por su parte, el comportamiento de las plantas dentro de los núcleos fue contrario al encontrado dentro del bosque, puesto que en los núcleos existía mayor disponibilidad de luz y por tal razón, las plantas presentaron un mayor crecimiento horizontal con el fin de ocupar mayor espacio (Cabrera y Ramírez, 2014).

La Figura 6 muestra las especies que son recomendables para iniciar el proceso de restauración dependiendo del disturbio. 


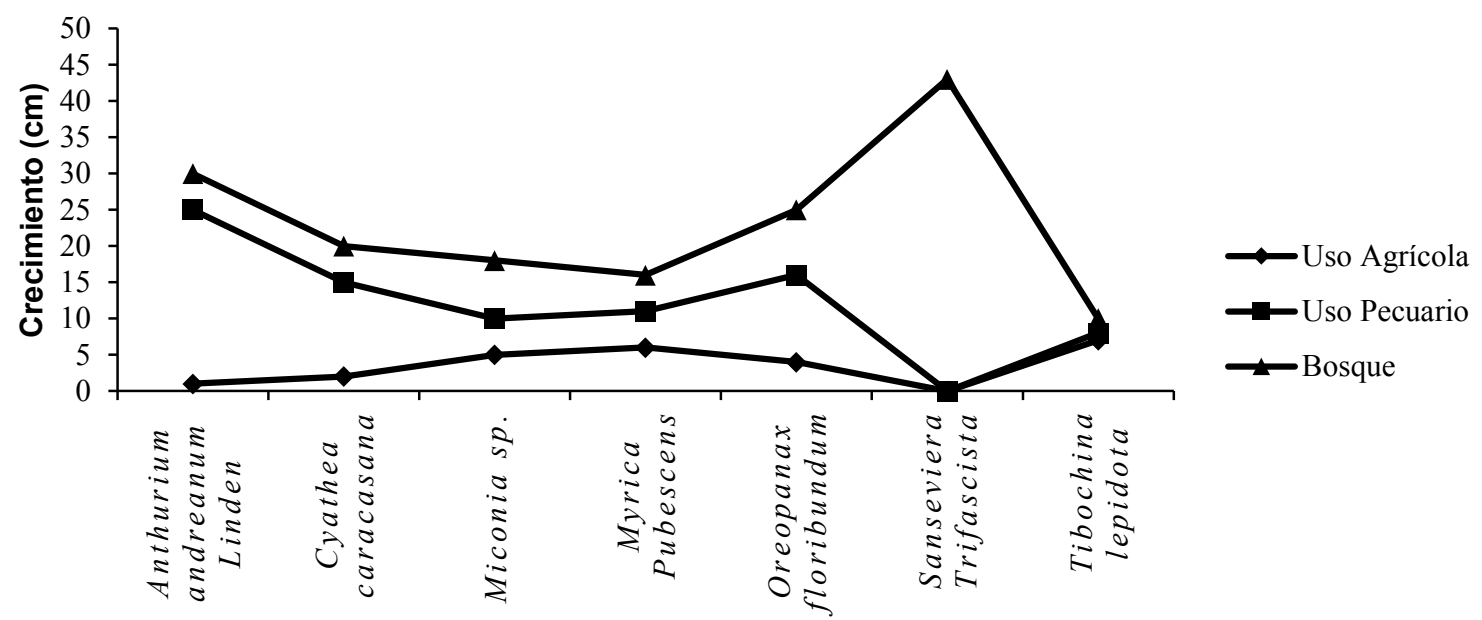

Figura 6. Especies potenciales para la restauración según los tres usos de suelo.

Teniendo en cuenta el uso de suelo agrícola, el cual ha sido sometido a varias etapas de disturbios por diferentes periodos de tiempo, se puede decir que hace parte de las muchas áreas en el mundo que están tan alteradas, que se deben emprender otras acciones como la rehabilitación, la reclamación 0 reemplazo y la revegetalización. En general, el concepto de restauración abarca o incluye actividades como la rehabilitación o reemplazo. En muchos casos, la plantación de árboles nativos o de especies pioneras dominantes y de importancia ecológica puede iniciar una rehabilitación (Vargas et al., 2010), la cual debe venir acompañada de otras estrategias para restaurar desde la composición del suelo, dependiendo de las deficiencias que éste presente según la historia de disturbios de la zona.

En consecuencia, la capacidad de restaurar un ecosistema dependerá de una gran cantidad de conocimientos, por ejemplo, el estado del ecosistema antes y después del disturbio, el grado de alteración de la hidrología, la geomorfología y los suelos, las causas por las cuales se generó el daño, la estructura, composición y funcionamiento del ecosistema preexistente, la información acerca de las condiciones ambientales regionales, la interrelación de factores de carácter ecológico cultural e histórico, es decir, la relación histórica y actual entre el sistema natural y el sistema socioeconómico, la disponibilidad de la biota nativa necesaria para la restauración, los patrones de regeneración, 0 estados sucesionales de las especies como son las estrategias reproductivas, los mecanismos de dispersión, las tasas de crecimiento y otros rasgos de historia de vida o atributos vitales de las especies; además, las barreras que detienen la sucesión y el papel de la fauna en los procesos de regeneración (Vargas et al., 2010).

De acuerdo con Herrera (2011), en un escenario antropizado, la transformación de los paisajes multifuncionales en territorios intensificados supone un beneficio económico a corto plazo, pero una pérdida de bienestar humano a largo plazo debido a la degradación de la biodiversidad y, por lo tanto, la pérdida de un flujo variado de servicios, así como la pérdida de valores culturales como la identidad cultural, el conocimiento ecológico local, o el sentido de pertenencia de muchas comunidades rurales.

\section{CONCLUSIONES}

La restauración ecológica de disturbios antrópicos para la zona Alto Andina implicó un conjunto de técnicas, las cuales se ajustaron al tipo de suelo degradado por la pérdida de cobertura. La 
Bacca y Burbano - Restauración ecológica de disturbios antrópicos.

aplicación de los mecanismos está en función de elementos entre los que deben considerarse: las características particulares del sistema a restaurar, la intensidad del deterioro, el objetivo del área, las especies involucradas y los resultados esperados.

Las variables que mas incidencia tuvieron para los tres tipos de uso del suelo analizados fueron para bosque, la altura de las plantas, para uso de suelo agrícola y pecuario, cobertura y número de ramas.

El uso de suelo agrícola presentó una menor tasa de crecimiento de especies potenciales para restauración, por lo tanto el suelo presenta un mayor tiempo de disturbio y deterioro que debe ser tratado con mayor rigurosidad.

Las especies que mejores resultados de crecimiento vertical y horizontal permitieron observar fueron $A$. andreanum paa uso de suelo bosque y 0 . floribundum en suelos bajo uso pecuario y 0 . floribundum en suelos de uso agrícola.

Conflicto de intereses: Los autores declaran que no hay conflicto de interés.

\section{REFERENCIAS BIBLIOGRÁFICAS}

Abreu, Z., Llambí, L. \& Sarmiento, L. (2009). Sensitivity of Soil Restoration Indicators during Páramo Succession in the High Tropical Andes: Chronosequence and Permanent Plot Approaches. Restoration Ecology. 17(5): 619-27.

Aidé, T.M. \& Cavelier, J. (1994). Barriers to lowland tropical forest restoration in the Sierra Nevada de Santa Marta, Colombia. Restoration Ecology. 2(4): 219-229.

Aranguren, A. \& Monasterio, M. (1997). Aspectos de la dinámica del nitrógeno en parcelas con diferente tiempo de descanso en el Páramo de Gavidia (Andes Venezolanos). Liberman, M. y Baied, C. (eds). Desarrollo sostenible en Ecosistemas de Montaña: Manejo de áreas frágiles en los Andes. 171-181 p. La Paz: The United Nations University (UNU).
Aronson, J., Blignaut, J. Milton, S. \& Clewell, A. (2006). Natural capital: the limiting factor. Ecological Engineering. 28(1): 1-5.

Barrera, J; Valdés, C. (2007). Herramientas para abordar la restauración ecológica de áreas disturbadas en Colombia. Universitas Scientiarum. 12(2): 11-24.

Barrera, J., Valdés, C., Moreno, A., Rondón, C., Montes, N., Sarmiento, E., Jiménez, J., Miranda, B., Corso, V., Cárdenas, T., Cotes, M. \& Clavijo, A. (2004). Restauración ecológica de la microcuenca Santa Helena, Suesca, Cundinamarca. Recuperada de http://sie.car.gov.co/bitstream/handle/20.500.11786/33690/10484. pdf?sequence $=1 \&$ isAllowed $=y$.

Bestelmeyer, B. T., Brown, J. R., Havstad, K. M., Alexander, R., Chavez, G., \& Herrick, J. E.. (2003). Development and use of state-and-transition models for rangelands. Journal of Range Management. 56: $114-126$.

Blignaut, J., Aronson \& Groot, R. (2013). Restoration of Natural Capital: A Key Strategy on the Path to Sustainability. Ecological Engineering. 65: 54-61. doi: https://doi.org/10.1016/j.ecoleng.2013.09.003

Bradshaw, A. (1987). Reclamation of derelict land and the ecology of ecosystems. En: William R. Jordan, III, William R. Jordan, Michael E. Gilpin, John D. Aber. Restoration ecology: a synthetic approach to ecological research. pp. 53-74. Inglaterra: Cambridge University Press. 342p.

Cabrera, M. \& Ramirez, W. (2014). Restauración ecológica de los páramos de Colombia: Transformación y herramientas para su conservación. Bogota: Instituto de Investigación de Recursos Biológicos Alexander von Humboldt (IAvH). 296 p.

Cantillo, E., Rodríguez, K. \& Avella, E. (2004). Diversidad y caracterización florística estructural de la vegetación arbórea en la Reserva Forestal Carpatos (Guasca Cundinamarca). Colombia forestal. 8(17): 5-21. doi: https://doi.org/10.14483/ udistrital.jour.colomb.for.2004.1.a01. 
Clewell, A.F. \& Aronson, J. (2006). Motivations for the Restoration of Ecosystems. Conservation Biology. 20(2): 420 - 428.

Constantino, E., Calderón E. \& Farfán J. (2006). Cattleya trianaeLinden \& Rchb. f Libro Rojo de Plantas de Colombia. Volumen 3. Bogotá, Colombia: Instituto Alexander von Humboldt - Ministerio de Ambiente, Vivienda y Desarrollo Territorial. $828 \mathrm{p}$

Etter A. (1998). Mapa general de ecosistemas de Colombia $(1: 2,000,000)$. En: M.E. Chaves y N. Arango (eds). Informe nacional sobre el estado de la biodiversidad en Colombia-1997. Bogotá, Colombia: Instituto de Investigación en Recursos Biólogicos Alexander von Humboldt.

Farrington, J. \& Martin, A. (1988). Farmer Participation in Agricultural Research: A Review of Concepts and Practices. Londres: Overseas Development Institute. 84p.

Fierro-Morales, J. (2012). Políticas mineras en Colombia. Bogotá: Instituto Latinoamericano para una Sociedad y un Derecho Alternativos ILSA. E.U.: Digiprint Editores. 264p.

Finegan, B. (1993). Procesos dinámicos en bosques naturales tropicales. Curso de bases ecológicas para la producción sostenible. Costa Rica: CATIE, Turrialba. $25 \mathrm{p}$.

Gentry, A. (1991). The distribution and evolution of climbing plants. En: Putz F.E. \& Mooney H.A. pp 3-52. The Biology of Vines. Cambridge: Cambridge University Press. 512p.

Grenier, L. (1998). Working with Indigenous Knowledge: A Guide for Researchers. Ottawa, Canada: International Development Research Centre - IDRC. . 115p.

Herrera, J. (2011). El papel de la matriz en el mantenimiento de la biodiversidad en hábitats fragmentados. De la teoría ecológica al desarrollo de estrategias de conservación. Ecosistemas. 20 (2): 21-34.

Hobbs, R. \& Harris, J. (2001). Restoration ecology: repairing the earth's ecosystems in the new millenium. Restoration Ecology. 9(2): 239246. doi: https://doi.org/10.1046/j.1526100x.2001.009002239.x
Holdridge, R. (1990). Zonas de vida de Colombia. Medellín: Universidad Nacional de Colombia, Sede Medellín. 81p.

Holl, K. \& Howarth, R. (2000). Paying for Restoration. Restoration Ecology. 8 (3): 260-267. doi: https:// doi.org/10.1046/j.1526-100x.2000.80037.

IAvH - Instituto Alexander von Humboldt. (2012). Portafolio Nacional de Restauración de Páramos y Humedales de Alta Montaña. Colombia: Convenio Interadministrativo (092), Ministerio de Ambiente y Desarrollo Sostenible. 260-267 p.

Jaimes, V. \& Sarmiento, L. (2002). Regeneración de la vegetación de páramo después de un disturbio agrícola en la Cordillera Oriental de Colombia. Ecotropicos. 15(1): 61-74.

Johnson, M. (1992). Lore: Capturing Traditional Environmental Knowledge. Ottawa: Diane Publishing. 200p.

Kattan, G. (2002). Fragmentación: patrones y mecanismos de extinción de especies. Ecología y conservación de bosques neotropicales. 1: 561582.

Lagos, T., Criollo, H. \& Mosquera, C. (2001). Evaluación preliminar de cultivares de uvilla o uchuva (Physalis peruviana L.) para escoger materiales con base en la calidad del fruto. Rev. Cienc. Agri. 18(2): 82-94.

Llambi, L. \& Cuesta, F. (2013). La diversidad de los páramos andinos en el espacio y en el tiempo. Venezuela: Instituto de Ciencias Ambientales y Ecológicas - ICAE, Universidad de los Andes. Consorcio para el Desarrollo Sostenible de la Ecorregión Andina (CONDESAN). 39p.

Machlis, G. (1993). Áreas protegidas en un mundo cambiante: Los aspectos cientificos. Caracas, Venezuela: Parques y progreso. UICN, BID. 37$53 p$.

Otavo, E. (1994). Análisis estructural de la vegetación. Bogotá: Ministerio del Medio Ambiente. OIMT. PNUD.10 p. 
Plan Nacional de Restauración. (2015). Restauración ecológica, rehabilitación y recuperación de áreas disturbadas. Colombia, Bogotá, D.C.: Ministerio de Ambiente y Desarrollo Sostenible. 92p.

Ramírez-Marcial N. (2014). Survival and growth of tree seedlings in anthropogenically disturbed Mexican montane rain forests. Journal of Vegetation Science. 14(6): 881-890. doi: https:// doi.org/10.1111/j.1654-1103.2003.tb02221.x

Rehm, G. (2009). Soil cation ratios for crops production. Estados Unidos de América: Minneapolis, Soil Science Department. University of Minnesota. 533p.

Rodríguez, B. \& Van Hoof B. (2004). Desempeño ambiental del sector palmero en Colombia: Evaluación y perspectivas. Bogotá D.C., Colombia: FEDEPALMA. 157p.

SER - Society for Ecological Restoration. (2004). The SER International Primer on Ecological Restoration. Washington D.C: Science and Policy Working group. $9 \mathrm{p}$.

Tejedor, N., Álvarez, E., Arango, C., Araujo, M., Blundo, C., Boza, T., La Torre, M.A., Gaviria, J., Gutiérrez, N., Jørgensen, P., León, B., López, R., Malizia, L., Millán, B., Moraes, M. Pacheco, S., Rey, J.M., Reynel, C., Timaná de la Flor, M., Ulloa, C., Vacas, 0., Newton, A. (2012). Evaluación del estado de conservación de los bosques montanos en los Andes tropicales. Ecosistemas. 21(1-2): 148-166.

Terborgh, J. (1992). Diversity and the tropical rain forest. New York: Scientific American Library. 243p.

UICN - Unión Mundial para la Naturaleza. (1999) Informe del undécimo Foro Global sobre Biodiversidad, Explorando la sinergia entre la Convención Marco de las Naciones Unidas sobre Cambio Climático y la Convención sobre Diversidad Biológica.Canada: UICN, Gland, Suiza y Cambridge, Reino Unido. 50p.

Vargas, O., Díaz, A., Trujillo, L., Velasco, P., Díaz, R., León, 0. \& Montenegro, A. (2007). Barreras para la Restauración Ecológica. Bogotá: Universidad Nacional de Colombia -Colciencias. 46-66p.
Vargas, R., Reyes, B., Gómez, R. \& Díaz, T. (2010). Guías técnicas para la restauración ecológica de ecosistemas convenio de asociación No. 22 entre Ministerio de Ambiente, Vivienda y Desarrollo Territorial (MAVDT) y Academia de Ciencias Exactas, Físicas y Naturales (ACCEFYN). Bogotá: Grupo de restauración ecológica (GREUNAL), Universidad Nacional. 136 p.

Vargas, R. (2011). Restauración ecológica: biodiversidad y conservación. Acta Biológica Colombiana. 16(2): 221-246.

Vargas, W. \& Ramírez, W. (2014). Restauración del bosque seco tropical en Colombia. El Bosque Seco Tropical en Colombia. Bogotá: Instituto de Investigación de Recursos Biológicos Alexander von Humboldt (IAvH). 221 - 246 p.

Walker, L. \& Del Moral, R. (2003). Primary Succession and ecosystem Rehabilitation. United Kingdom: Cambridge University Press. 442p.

Whisenant, S.G. (1999). Reparing damaged wildlands: A process oriented, landscape-scale approach. Cambridge: UK. Cambridge University Press. 328p.

Whitmore, T. (1975). Tropical Rain Forest of the Far East. London: Oxford Univ. Press. 16 - 18 p. 